ROCZNIKI HUMANISTYCZNE

Tom LXIX, zeszyt 5 - 2021

DOI: http://doi.org/10.18290/rh21695-7

MAREK JAKUBÓW

\title{
„GESETZT, DU KENNTEST DEN TITEL DES BILDES NICHT“: ZU NAVID KERMANIS UMGANG MIT SAKRALEN BILDERN
}

\begin{abstract}
A bstract. Kermanis aufsehenerregendes Buch Ungläubiges Staunen. Über das Christentum (2015), das in den letzten Jahren 14 Auflagen erlebte, ist in erster Linie ein Versuch, eine neue religiöse Sprache zu schaffen. Mit deren Hilfe sucht er die vorherrschende Tendenz zu überwinden, die Bruno Latour „Bildstürmerei“ nennt. Sie sei natürliche Konsequenz der bisherigen rationalistischen Religionskritik, die zur Folge hat, dass das religiöse Sprechen entweder an Bedeutung verloren hat oder als exklusives Phänomen behandelt wird. Indem Kermani auf die vorwiegend barock-katholische Bildtradition zurückgreift, entwickelt er eine Schreibstrategie, die zum Ziel hat, die bekannten religiösen Bilder, zunächst von bestehenden konfessionellen und profanästhetischen Deutungen zu befreien. In einem die Grenze zwischen den zwei Medien überschreitenden Annäherungsprozess bemüht er sich dann, ihnen verborgene Inhalte abzugewinnen und sie in einer für die Gegenwart adäquaten Sprache wiederzugeben, die in der Erfahrungs- und Vorstellungswelt des modernen Menschen fest angesiedelt ist.
\end{abstract}

Schlüsselwörter: Navid Kermani; Wort und Bild; Intermedialität; deutschsprachige Literatur; religiöse Rede.

Auf den ersten Seiten seines 2015 veröffentlichten Buches Ungläubiges Staunen. Über das Christentum lässt Navid Kermani seine Leser zunächst über die Identität des Erzählers rätseln. Eins steht fest, er ist ein Rom-Reisender und begibt sich in das Kloster auf dem Monte Mario, um dort - wie er sagt - ein Bild der „Jungfrau“ (Kermani 9) zu sehen. Im Gegensatz zu seinem „katholischen Freund“1, der einen Aufsatz über den Evangelisten Lukas

Univ.-Prof. Dr. habil. MAREK JAKUBÓw - Katholische Johannes-Paul-II.-Universität Lublin, Institut für Literaturwissenschaft, Lehrstuhl für deutschsprachige Literatur und Kultur; Korrespondenzadresse: Al. Racławickie 14, 20-950 Lublin; E-Mail: marek.jakubow@kul.pl; ORCID: https://orcid.org/0000-0003-1024-0502.

${ }^{1}$ In einem Interview suggerierte Kermani, dass diese fiktive Gestalt die Züge von Klaus Berger und Martin Mosebach sammelt (vgl. Interview mit Navid Kermani). 
- den vermeintlichen Autor des Werkes geschrieben hat und ihn bei der Besichtigung begleitet, wie auch zu den Nonnen, die in der Nähe des ihnen vertrauten Bildes nach gewohntem Rhythmus leben, offenbart er immer wieder eine überraschende Unkenntnis des religiösen Kontextes. Die kunsthistorischen Angaben zum Bild sind für ihn sekundär. In den Vordergrund rückt die Unmittelbarkeit des persönlichen Innehaltens. Wenn er das Bild sieht, fühlt er sich von ihm unerwartet angesprochen: „Die Jungfrau hat mich angeschaut, ohne Alter" (Kermani 9).

Als sich in einem weiteren Kapitel herausstellt, dass der Betrachter ein Muslim ist, scheint das Rätsel geklärt zu sein ${ }^{2}$. Die spontane Assoziation mit dem schon im Titel angeführten Ungläubig-sein, das aus religiösen und kulturellen Unterschieden herrührt und sich im Vorstellungskomplex des Fremden verdichtet, erweist sich jedoch als eine Falle für einen durch die europäisch-christliche Kultur geprägten Leser. Der Erzähler wurde ,im protestantischen Siegen geboren" (Kermani 258) und in der Bundesrepublik Deutschland der 70er und 80er Jahre sozialisiert. $\mathrm{Zu}$ den prägenden Momenten seiner Biografie gehören - wie er bekennt - die Friedens- und Umweltbewegung wie auch die Lektüre Adornos. Darüber hinaus fährt er in die italienische Hauptstadt mit Hölderlins und Jean Pauls Texten im Rucksack, die - worauf die zitierten Stellen aus ihren Werken hinweisen - die säkulare Zeit wie auch die moderne religiöse Sprachlosigkeit vorwegnehmen. Sein anfänglich mangelnder Bezug zu dem Bild erfolgt demnach nicht nur aus seiner Zugehörigkeit $\mathrm{zu}$ dem bilderlosen Islam, sondern auch aus seinem deutschen, durch protestantische Tradition geformten, bilderarmen Umfeld.

Die Unfähigkeit des Betrachters, sein unerwartetes, aber bleibendes Erlebnis in eine kommunizierbare Sprache zu transformieren, lässt ihn nach schon überlieferten Arten der Bildbetrachtung greifen. Die Klausur der barocken Kirche, in der nur die rhythmischen Gebete der verschleierten Nonnen zu hören sind, und die sich vor dem dunklen Hintergrund deutlich abzeichnende Ikone mit dem „Gesicht Mariens in erstaunlichsten Farben“ (Kermani 10) öffnen zwangsläufig den intertextuellen Bezug zu Wackenroders Herzensergießungen eines kunstliebenden Klosterbruders und zu der Folie des romantischen Kunsterlebnisses. Nichtsdestoweniger ist für Kermanis Kunstbetrachter der Weg zu einer derartigen „stillen Entzückung“ (Wackenroder 167) versperrt. $\mathrm{Zu}$ aufdringlich sind die Gedanken über die katholische Hierarchie oder das Dogma der Jungfräulichkeit.

\footnotetext{
${ }^{2}$ In den meisten Kommentaren wird das Buch aus der Perspektive der interkulturellen Gegenwartsliteratur in Deutschland behandelt (vgl. Hofmann 13-16).
} 
Sein Mitteilungsdefizit ist auch nur schwer mit der mangelnden Begrifflichkeit zu erklären, weil sie ihm doch im Übermaß zur Verfügung steht. Er erweist sich als Kenner der Bibel und Marienmystik gleichermaßen wie von Apokryphen, Hagiographien und unterschiedlichen diesbezüglichen kunsthistorischen und literarischen Werken. Sie liefern zwar informatives Quellenmaterial, scheinen aber für seine Narration nur bedingt von Nutzen zu sein. Es wird somit eine Perspektive des Verstummens angenommen, dem die schon von Hölderlin in Die scheinheiligen Dichter thematisierte Säkularisierung des Religiösen und der Verlust der emotionalen Bindung an die sakralen Darstellungen wie auch der adäquaten Sprache zu ihrer Beschreibung zugrunde liegen.

Kermani teilt diese Erkenntnis mit Bruno Latour, der in seinem predigthaften Essay Jubilieren. Über religiöse Rede (2002) diesen Mangel in der modernen Gesellschaft auf die konsequent geübte Religionskritik zurückführt: „Der Hammer der radikalen Kritik ist zurückgeprallt; uns hat er an der Stirn getroffen; uns hat er stumm und sprachlos gemacht" (Latour 209). Die alten Vorstellungen wurden abgeschafft und durch Substitute ersetzt, die keinen Anspruch auf Verbindlichkeit stellen. Parallel verlaufen die Entwicklungen in der bildenden Kunst, wo das Bild jeglichen Bezug zum Realen oder Metaphysischen verloren hat und nach Jean Baudrillard zu einem Simulacrum wurde (vgl. Bator 64-74). Hans Belting schreibt über den Schwund des Vertrauens zu dem Bild und nennt dieses Phänomen den „modernen Ikonoklasmus“ (Strehle 515).

Latours Suche nach einer neuen religiösen Rede führt nicht über die Herstellung von festen Referenzen. Er versteht die negativen Reaktionen, Ängste vor der Wiederkehr der traditionellen Religionen als „eine[r] totale[n], oft totalitäre[n] Lebensform auf der Suche nach einer vollkommenen Welt" (Latour 216) und lehnt jegliche Verbindung der Religion mit pragmatischpolitischen Inhalten ab. Dabei unterstreicht er die Gegenwartsbezogenheit der modernen religiösen Rede:

Um von Religion zu sprechen, gibt es keine bessere Welt als die unsere, diese hier, die einzige, die wir haben, da sich an diese gegenwärtige Welt in seiner eigenen Sprache wenden muß, wer will, daß die Worte der Überlieferung von neuem danach klingen, daß sie der Wahrheit entsprechen. Darauf warten, daß uns ein Wunder in andere Zeiten und an andere Orte entführe, um wahrhaftig zu reden, heißt einer lügenhaften Konstruktion aufsitzen. (Latour 217)

Diese Konstatierungen korrespondieren sehr gut mit der von Kermani im Interview mit Tobias Haberl geäußerten Meinung, dass sich die fundamenta- 
listischen Befürworter der Wiederkehr zur Tradition in Wirklichkeit gegen die Tradition wenden. Darüber hinaus ist für ihn die „Religion niemals das göttliche Wort pur. Sie ist das sich stets verändernde Verhältnis von Menschen zu diesem Wort" (Haberl).

In den Vordergrund tritt bei Latour das Relationale der religiösen Sprache und ihre emotionale Ladung. Er vergleicht sie mit der „Vergegenwärtigung“ der Liebe zwischen zwei Liebenden. Sie drückt zwar nicht alle ihre Existenzformen aus, fügt aber diesem Phänomen ,ihre eigene Tönung hinzu, ohne die niemand dem anderen nahe wäre, ohne die die Zeit sich nicht erfüllen würde, ohne die die Geschichte vergebens verstriche, ohne die der Tod triumphiere" (Latour 219). Sie annihiliert die alten Redeweisen nicht, die der katholischen Tradition mit allen ihren negativen Erscheinungen als Reservoir der Wahrheit und Fehler (vgl. Latour 235) angehören, sondern übersetzt sie in eine neue Rede. Ihr Nutzen besteht für ihn nicht mehr in der Vermittlung einer Botschaft oder Doktrin. Es handelt sich um „ein re-präsentationales Sprechen, das immer wieder aufs Neue den Anlauf nimmt, den Glauben im Fall der religiösen Rede oder die Liebe im Fall der Liebesrede zu erneuern und wiederherzustellen“ (Müller und Grizelj 190).

In diesem Zusammenhang ist Kermanis Zuwendung zu dem sakralen Bild in Ungläubiges Staunen als Versuch anzusehen, das Vertrauen zu ihm wiederzugewinnen ${ }^{3}$, d.h. eine in ihm eingefangene religiöse Erfahrung hervorzukehren und in einer adäquaten Sprache ausdrücken zu können, die ein aufs Neue erwachtes inniges Verhältnis des modernen Betrachters zu ihm wiedergeben könnte. Wenn er in einem Band Texte und Bilder zusammenstellt, suspendiert er die geltende, in solchen Konstruktionen zeit- und kontextbedingte klare „Vorverständigung“ (Schmitz-Emans 10) und stellt somit die Codes, welche das Miteinander von Texten und Bildern regeln, in Frage. „Dies bedeutet - schreibt Monika Schmitz-Emans über eine solche Beziehung -, „daß gerade die literarischen Texte, die sich auf Bilder beziehen, dazu provozieren, über das Sagen und Zeigen, das Sprechen und Schweigen der Bilder nachzudenken“ (Schmitz-Emans 10). Kermani zieht Kataloge, Lexika, Alben, Reiseführer, wissenschaftliche Abhandlungen heran, die ebenso wie die inszenierten Räume mit Lichteffekten, in denen er die Werke

\footnotetext{
${ }^{3}$ In der deutschsprachigen Literatur der letzten Jahre vollzieht sich eine bemerkenswerte Widergewinnung des Vertrauens zu dem Bild. Malte Dominik Krüger macht die Sensibilität für Bilder auch zum entscheidenden Moment für die religiöse Erfahrung des modernen Menschen. Der spätmoderne Protestantismus wird nach ihm als „kritische Bildreligion verständlich und dabei ein Bild Christi präsentiert, das einerseits als solches identifizierbar ist und andererseits sich zeitgemäß in einer von Bildern dominierten Kultur darstellt“ (Krüger XIV).
} 
betrachten kann, in einem kulturellen oder ideologischen Gerüst fest verankerte, zuweilen vereinfachte Interpretationsmuster bieten. Er ist zum Beispiel verblüfft, wenn er bei näherer Betrachtung des Bildes Hiob von Dürrer, das er zunächst auf dem Laptop gesehen hat, statt der Jauche klares Wasser entdeckt. Hiobs Frau verspottet seinen Gatten nicht und „blickt keineswegs hämisch [...] wie es aber doch auch die CD-Rom ausdrücklich erklärt" (Kermani 106). Künstlich findet er das Foto mit Renis Kreuzigung, das etwas von den billigen Ansichtskarten hat, die die Zigeuner vor der Kirche San Lorenzo in Rom an Touristen verkaufen, oder befremdend wirken die sakralen Sammlungen in dem heutigen Köln, ,in dem das Heilige nur noch Reminiszenz oder schon Weltkulturerbe ist" (Kermani 251). Die bestehenden, profanen Deutungen werden von Kermani zwar als Beschreibungsmodi und Wissensbestände anerkannt, zugleich aber relativiert:

Wie der Leser bereits auf den ersten Seiten bemerkt hat, erhebt das Buch keinen wissenschaftlichen Anspruch, sondern ist eine frei assoziierende Meditation - ein Staunen eben - über vierzig Bilder und Begriffe, Heilige und Rituale. [...] Nicht nur ist mein Buch an der geglaubten und auch ästhetischen Wahrheit meist interessiert als an dem, was heute für historisch wahr gehalten wird; es ist selbst Ausdruck eines religiösen und ästhetischen Erlebens. (Kermani 292)

Da das Bild dem Leser in Form einer Reproduktion zugänglich ist, wird der Text nicht nach dem Prinzip der Evidentia eingesetzt, um ihm die beschriebenen Gegenstände und Handlungen „optimal vor Augen zu stellen“ (Rosen 171), und muss somit die Funktion der rhetorischen Ekphrasis nicht erfüllen. Als Kommentar wird er zum Element einer Schreibstrategie (vgl. Markowski 229), deren Eigenart in der Überschreitung der Grenze zwischen den beiden Medien besteht. Er wirft zwar eine Betrachtungsperspektive auf, indem er nur auf einige Aspekte aufmerksam macht. Gleichzeitig dient das Bild aber in seiner Materialität, das in die Beschreibung integriert wird, der Erweiterung seiner rein sprachlichen Dimension. Die ganze Struktur bekommt auf diese Weise den Anstrich des Emblematischen.

Nicht die ganze Geschichte in allen Facetten, die ein Bild erzählt, wird in Kermanis Narration wiedergegeben. Viel wichtiger ist eine Geste oder ein Blick, mit einem Wort ein Detail, das den Weg zu einer bisher nicht exponierten Sichtweise öffnet. Es ist eine quasi anamorphotische Annäherung an das Dargestellte, die dem Leser erlaubt, es aus einem neuen, ungewöhnlichen Blickwinkel wahrzunehmen (vgl. Maffei und Fiorentini 92-94) und zu einer Erkenntnis zu gelangen, die bisher durch die etablierten Deutungen 
verdeckt, verdrängt oder marginalisiert wurde. Das Bild ist für ihn keine Illustration eines Theologems oder einer moralischen Lehre. Kermani verwandelt es auch nicht nach den Lessingschen Regeln in eine Handlung, sondern intensiviert das Gesehene und Erlebte mit Hilfe aussagekräftiger Redemittel und bezieht es auf zwischenmenschliche Relationen, denen er den Charakter einer anthropologischen Konstante verleiht. Die Titel der einzelnen Kapitel heißen Mutter, Sohn, Erniedrigung, Freundschaft und rufen grundlegende menschliche und religiöse Erfahrungen wie Liebe, Lust, Tod oder Berufung auf den Plan.

In einer persönlichen Auswahl von Kunstwerken richtet Kermani seine Aufmerksamkeit in erster Linie auf suggestive Beispiele der religiösen Malerei des katholischen Barocks. Ihre Wiederentdeckung wird von ihm sogar mit der Wiederentdeckung des Religiösen schlechthin gleichgesetzt (vgl. Navid Kermani). Seinen Umgang mit dem Bild veranschaulicht die Beschreibung des Besuchs in Santissima Trinità dei Monti. Die Aufmerksamkeit des erkälteten Erzählers, der sich die „anamorphotischen Fresken“ (Kermani 204) anschaut, „fesselt ein Detail in der Mitte der Querwand“ (Kermani 205). Es ist „der Mundschenk vor dem Tisch, an dem Jesus Christus mit Maria speist" (Kermani 205). Er befindet sich an der Grenze zwischen zwei Welten: „Der Rumpf des Mundschenks ist zu Jesus gerichtet, aber mit dem Kopf und der Karaffe wendet er sich zum Saal, wo die Mönche aßen“ (Kermani 205). Die Verlängerung der Bildwirklichkeit in die Welt des Klosters kam vor Jahren auch durch „eine kleine Treppe hinauf zum Bild“ (Kermani 205) zustande. Die beiden Bereiche verbindet eine zweitrangige Gestalt, die dem Betrachter eine in seiner unmittelbaren Wirklichkeit verankerte Erfahrung nahelegt:

[...] der Mundschenk, der aus der Wand tritt, scheint als einziger eine Funktion zu haben, eine Botschaft auszudrücken, eine sehr katholische: Brüder, laßt es euch schmecken! Es ist Blut von Seinen Bluten, und zwar nicht sakral aufgeladen wie nebenan in der Messe, nicht als Sakrament, Realpräsenz, Gott-Essen, vielmehr so sinnfällig wie augenzwinkernd. (Kermani 205)

Den Betrachter interessieren in erster Linie „Gott der Mensch“ (Kermani 64) und Christi Nachfolger, die innere und äußere Erfahrungen intensiv ausleben. Es beeindrucken ihn in Rom „zuerst die Kreuzigungen, Verbrennungen, Ermordungen und Martyrien" (Kermani 123) und dann aber auch allägliche Situationen, in denen das Gelebte deutlich zutage tritt. Wenn er auf Caravaggios Die Hochzeit zu Kana vorsichtig zukommt, was das jeden 
Einschnitt eröffnende „vielleicht" signalisiert, ist das panoramaartige Bild vor allem ein Hintergrund, vor dem er unter zahlreichen Gestalten die kaum sichtbare Figur Christi wahrnimmt, der hier aber nicht in Hinsicht auf seinen großen Erlösungsauftrag, sondern als ein gut aussehender junger Mann, der „so viel Freude am Essen und Trinken [hatte]“ (Kermani 21), herausgefischt wird. Die Kreuzigung Petri, die man „von unten aus einem seitlichen Winkel, also eingeschränkter Perspektive betrachten kann“, ist „plastisch“, „lebendiger als das Leben“ und „springt aggressiv ins Auge“ (Kermani 123). In den Vordergrund tritt die pure menschliche Angst (vgl. Kermani 127). Auf dem Bild von El Greco Der Abschied Christi fasziniert den Erzähler ein kurzer Blick, der eine tiefe innige Bindung zwischen den zentralen Figuren Christi und Marias andeutet: „El Greco, so scheint mir, hat sich gewundert, als er ihre Lippen so sinnlich malte, daß sie sich jeden Augenblick zu einem Kuß vereinigen möchten. Er hat zwei Blicke hinzugefügt, die in den Evangelien fehlen und doch jeder erinnern müßte, der je groß geliebt“ (Kermani 37). Judith ist keine pathetische Heldin. Sie ähnelt eher der Hebbelschen Protagonistin, die sich als gekränkte Frau von ihrer Rachelust leiten lässt (vgl. Kermani 113).

Die Sinnlichkeit der Darstellung manifestiert sich auch in der Ausdruckhaftigkeit und Eindrücklichkeit der Sprache (vgl. Laak 3). Kermani sieht in den Jesus-Bildern eine wirklichkeitsnahe Gestalt, wie das in der bildenden Kunst zum Beispiel Max Liebermann tat, als er seine „realistische Darstellung des Jesusknaben“ (Faass 7) malte. In seinem Text wird diese Perspektive radikalisiert, indem dem spontanen, unbefangenen Empfinden, das zum Beispiel salopp-direkte Hinweise der Wärter im Bode-Museum in Berlin andeuten (,zum Dickerchen den Korridor lang“ (Kermani 15), freier Lauf gelassen wird. Die kleine Jesusfigur ist einfach „häßlich“ (Kermani 14). Nicht die konventionellen Attribute des Heiligen treten in den Vordergrund, sondern die auffallende Körperlichkeit: ,[...] dieser offene Mund: hasenschartig der Unter-, hervorstehend der Oberkiefer, und mehr noch die Lippen: die untere kurz oder genaugenommen nicht kurz, sondern gestaucht, fett in die beiden Wölbungen sich dehnend, dazu die Oberlippe wie ein Zelt [...]“ (Kermani 14). Auf dem Bild von El Greco ist Jesus „,der Mann erst Anfang, Mitte zwanzig, die Stirnen faltenlos, die Wangen rosig, die Lippen samtweich wie bei Kindern und zugleich sinnlich gewölbt" (Kermani 32).

Die auf den ersten Blick radikale und vereinzelt pietätlose Ausdruckweise hat nicht zum Ziel, die behandelten Objekte zu degradieren. Sie bringt vielmehr Sichtweisen zur Geltung, die tabuisiert, verschwiegen oder für unange- 
bracht gehalten werden, und schafft die Distanz ab, die den Betrachter von dem musealen Exponat trennt. In den Bemerkungen zu Rembrandts Die Auferweckung des Lazarus wird schon am Anfang das Staunen gegenüber einer unerwarteten Situation exponiert, das er nicht nur in den dargestellten Figuren, sondern auch in sich selbst entdeckt. Er fühlt sich in die menschliche Trauer Christi um den Freund Lazarus ein: „Auch ich wünsche mir, spreche den Wunsch in eingestandener Selbstsucht gelegentlich aus, so Gott will vor meiner Frau und vor unseren Kindern zu sterben, damit mir nicht die Augen an ihrem Grab übergehen“ (Kermani 31). Die Christi-Gestalt selbst auf dem Bild von Veronese ist mit den ihm bekannten generationsbedingten Klischees (als Hippie oder „Gründungsmitglied der Grünen“ (Kermani 21) nicht zu vereinbaren und es ärgert den Erzähler, dass er mit seiner persönlichen Erfahrung vereinsamt ist und sie in geläufigen Kategorien nicht mitteilen kann, „weil es mir aussichtslos schien und ich vielleicht selbst für verrückt gehalten worden wäre, für einen der Spinner, die in der Fußgängerzone vorm Weltende warnen“ (Kermani 26). Er sieht darüber hinaus eine Parallele zu seinen eigenen Empfindungen, wenn er die Szene aus Caravaggios Tod Mariae auf das eigene Bangen um seine Mutter überträgt. Zuweilen lässt er sich sogar von seinen Gefühlen fortreißen. Es schockiert ihn zum Beispiel die unmenschliche Haltung Judiths, deswegen macht er mit Hilfe der Invektive seiner Empörung Luft, indem er „Blöde Kuh!“ (Kermani 116) ruft.

An manchen Stellen machen ihm seine eigenen Emotionen bewusst, wie dünn die Grenze ist, die ihn von der Gotteslästerung trennt (vgl. z.B. Kermani 15, 20). Dabei will er sich von naturalistischen oder bloß auf die Steigerung von Emotionen konzentrierten Darstellungen abgrenzen. Die angeführten veristischen Details, die Kermani den „derben Realismus“ (Kermani 124) nennt, sind aber keine für die Epoche typische Staffage, wie das der in seinem Kommentar zu Caravaggios Die Kreuzigung Petri zitierte Jakob Burkhardt sehen wollte, um dem objektiv Schönen zum Durchbruch zu verhelfen. Das Drastische, Brutale und sogar Ordinäre auf dem Bild steht nach Kermani im Kontrast zu den melodramatischen Narrationen und „[tritt] gerade dort am stärksten, [hervor], wo sich das Heilige oder die Liebe tatsächlich ereignen" (Kermani 124). Deswegen ist die Martyriumszene nicht nur sachlich, symbolisch, ästhetisch oder religionshistorisch, sondern vor allem „ergreifend“ (Kermani 124). Caravaggios Petrus „stirbt wie ein Mensch: ratlos, einsam, überrascht" (Kermani 124).

In den Kapiteln Lust I und Lust II, in denen er sich mit den Bildern Der Liebeszauber eines Niederrheinischen Meisters und Die Begegnung 
Joachims und Annas an der Goldenen Pforte von Giotto beschäftigt, verdrängt er das Erotische und Sexuelle, das stellenweise vulgär anmutet, aus den religiösen Bildern nicht, sondern betrachtet diese Komponenten als mit dem Heiligen vereinbarte Sphären. Im ersten Fall folgt der detaillierten Beschreibung der „Haltung der beiden Körper, die obszön zu nennen wäre“ (Kermani 252) die überraschende Feststellung, dass das Bild „unverfroren zwischen zwei Kruzifixen hängt" (Kermani 254). Sie deutet auf die Dissonanz in der Wahrnehmung des modernen Menschen hin, der die Sphären des Heiligen und Erotischen strikt voneinander getrennt hält und über keine passende Sprache verfügt, die eine solche Verbindung wiedergeben könnte, ohne sie in Frage zu stellen. Joachim und Anna sind ,im heutigen Westen Europas, in dem sich alle alles allerorten herauszunehmen scheinen, [...] zwei ungeniert knutschende Alte" (Kermani 255). Als Alternative liefert Kermani eine Beschreibung, in der sich die stilistisch durch eine Klimax verstärkte Freude über die Wiederbegegnung nach längerer Trennung voneinander, Zärtlichkeit, Zuwendung, langjährige Erfahrung des Zusammenlebens, Achtung und Begierde verdichten:

Wenn sie nach so vielen Jahren des gemeinsamen Alltags, des Streits, den es doch sicher gegeben haben wird, des Zorns, der Enttäuschung, der Zweifel, der Krisen, der Gebrechen und überhaupt des körperlichen Verblühens, der Fältchen und Falten, um nur das Geringste zu nennen - wenn zwei auch die Langeweile geschmeckt haben, die noch die leidenschaftlichste Liebe zu vergiften vermag [...]wenn zwei jede Pore, jeden nächtlichen Laut und jede Ausdünstung des anderen kennen und dennoch mehr als nur zugetan, nämlich zärtlich geblieben sind, sich ungeachtet ihres Alters noch immer so sehr begehren [...] (Kermani 256)

In den darauffolgenden Sätzen stellt er einen Bezug zu der Schilderung der intimen zwischenmenschlichen Beziehung in der Bibel her, die durch ein Zitat aus dem Hohelied bekräftig wird. Auf diese Weise schließt er sich einem schon vorhandenen Traditionsstrang an und modifiziert bloß das überlieferte Vorbild.

Die Beschreibung der einmaligen und persönlichen Empfindung von Nähe bei der Bildbetrachtung wird in die latent mitschwingende Struktur des mystischen Erlebnisses eingebettet, in dem „Lust und Gebet, Sex und Gott sich eins fühlen" (Kermani 154). Dem hagiographisch tapferen und ungerührten Darstellen zieht Kermani die Verbindung von Schmerz und lustvoller Erregung vor, die er in den Schriften des Heiligen Franziskus findet, in denen solche Begriffe wie „tiefer Genuß“ (vgl. Kermani 150), „Begierde“ 
und „Süße des Mitgefühls“ zentral sind. Als Parallele wird die Ekstase der Heiligen Theresa angeführt, die von Gian Lorenzo Bernini „explizit sexuell dargestellt“ (Kermani 151) wurde. Er grenzt sie aber von der ,alltäglich gewordene[n] Pornographie“ (Kermani 259) und ihrer selbstbezogenen, mit dem Sadomasochismus verwandten Variante in den erotischen Romanen ab, die „den Schmerz heutzutage zelebrieren“ (Kermani 156). Die physische Qual auf den sakralen Bildern wird immer mit dem persönlichen Ringen um das eigene Ich, „das sich verliert“ (Kermani 156), und dem „Dienst an den Menschen" (Kermani 155) gekoppelt.

Die Betonung der intensiven Farben, die in erster Linie als ein der Malerei vorbehaltenes Mittel gelten und zu einer ,wechselseitigen Erhellung der beiden Künste“ (Cheon 12) beitragen, stärkt die Aussagekraft des Textes. Auf dem Bild von El Greco Abschied Christi sind sie „licht und warm, das leuchtende Blau des Himmels, um Jesu Kopf der Heiligenschein, der wie gesagt auch Sonnenlicht sein könnte [...] dazu das volle Rot und Moosgrün ihrer Gewänder [...]“ (Kermani 36), Lazarus trägt „deutliche Züge der Verwesung und phosphoresziert auch noch leichengrau“ (Kermani 27).

Wenn Kermani das Sinnliche, Prägnante, Suggestive und Menschennahe zu Merkmalen der religiösen Sprache und Bildlichkeit macht, sucht er nach Begründung dafür nicht nur in den ihm geläufigen Alltagserfahrungen, sondern auch in theologischen Quellen, die sich der menschlichen Natur Christi zuwenden. Es sind unter anderem das „realistische“ (Kermani 18) Kindheitsevangelium nach Thomas, das die Lücke in der theologisch vertretbaren Jesusbiografie füllt und die homogene, verklärende Erzählung um das Element des Hässlichen ergänzt („Schönheit [wird] auch erst mitsamt ihrem Gegensatz wahr" (Kermani 19)), Origenes oder die Arianer. Hinzu kommt die katholische Realpräsenz und deren Folgen für die Wahrnehmung des Menschen in seiner Körperlichkeit, die auf den barocken Bildern in die Augen sticht, aber für den Bildbetrachter nicht selbstverständlich ist: „schließlich bin ich im protestantischen Siegen geboren und nicht im katholischen Rom“ (Kermani 258).

Die intensive Auseinandersetzung mit religiösen Bildern führt zu keinem radikalen weltanschaulichen Umbruch bei Kermanis Erzähler. Nach dem Kirchenbesuch in Santissima Trinità dei Monti folgt kein Paulus-Effekt: „keine Erleuchtung, keine Bekehrung, nicht einmal Läuterung, nur Frieden, der nach den zwei Stunden noch ein wenig anhielt" (Kermani 74). Die eigentliche Wende vollzieht sich, wenn er die zeitlich und kulturell bedingte 
Distanz überwindet, die ihn von den betrachteten sakralen Bildern trennt, und zur eigenen Sprache findet, mit deren Hilfe er über seine Faszination und emotionale Anteilnahme an überzeitlichen Erfahrungen berichten kann, die in verschiedenen Epochen und Kulturen dem Menschen zuteilwerden. Darauf weisen sowohl das Kapitel über den modernen Heiligen Pater Paolo Dall'Oglio mit dem beigefügten Videobild als auch über das Kloster Mar Musa mit dem Photo und den Anspielungen auf den Sufismus hin. Die Bildbetrachtung wird somit nicht ausschließlich Sache der subjektiven Wahrnehmung, was seit Descartes und der Philosophie des 18. Jahrhunderts zur Selbstverständlichkeit geworden ist (Hyman 33). Darüber hinaus wird ihr rein diskursiver Charakter überwunden, indem die Darstellungen selbst als materielle Zeugnisse in den Text mit eingebracht werden und die Grenze zwischen den beiden Medien flüssig gemacht wird. Das Spiel zwischen Unsichtbarem und Sichtbarem geschieht nicht ausschließlich in der Einbildungskraft des Betrachters. Das Sichtbare wird nicht annihiliert, sondern beglaubigt das Wort, das in seiner Vorstellungswelt verankert ist und ein sich anbahnendes, intensives, emotionales und vor allem menschennahes Erlebnis ausdrückt.

\section{LITERATUR}

Bator, Andrzej P. „Jean Baudrillard i problem obecności.“ Dyskurs, Nr. 4, 2006, 64-74, https://www.asp.wroc.pl/dyskurs/Dyskurs4/AndrzejPBator.pdf. Abruf am 14.5.2020.

Cheon, Hyun Soon. Intermedialität von Text und Bild bei Alexander Kluge. Zur Korrespondenz von Früher Neuzeit und Moderne. Königshausen \& Neumann, 2007.

Dalferth, Ingolf U., und Philipp Stoellger, Hrsg. Krisen der Subjektivität: Problemfelder eines strittigen Paradigmas. Religion in Philosophy and Theology. Mohr Siebeck, 2005.

Euler, Ralf, und Stefan Toepfer. „Koch entschuldigt sich bei Kermani“. FAZ, 27.11.2009. https:// www.faz.net/aktuell/rhein-main/hessen/hessischer-kulturpreis-koch-entschuldigt-sich-beikermani-1881561.html. Abruf am 6.7.2020.

Faass, Martin, Hrsg. Der Jesus-Skandal. Ein Liebermann-Bild im Kreuzfeuer der Kritik. MaxLiebermann-Veranstaltungs GmbH, 2009. http://www.liebermann-villa.de/docs/ attachments/b16d7df4-f4dc-4a1b-933f-a639b3ac1581/jesus-skandal-Web.pdf. Abruf am 3.7.2020.

Haberl, Tobias. „Natürlich ist Religion erst mal Pflicht.“ Süddeutsche Zeitung, 01.09.2015, https://sz-magazin.sueddeutsche.de/leben-und-gesellschaft/natuerlich-ist-religion-erstmal-pflicht-81594. Abruf am 19.03.2020.

Hoff, Gregor Maria. „Vor dem Kreuz: Blasphemische Inversionen.“ Kann man Gott beleidigen? Zur aktuellen Blasphemie-Debatte, hrsg. von Thomas Laubach, Herder, 2013, S. 75-90.

Hofmann, Michael. „Aus literaturwissenschaftlicher Perspektive“. Navid Kermani, hrsg. von Michael Hofmann, Klaus Stosch, Swen Schulte Eickholt, Königshausen \& Neumann, 2019, S. 13-16. 
Hyman, John. „Bilder betrachten.“ Bild - Bildwahrnehmung - Bildverarbeitung. Interdisziplinäre Beiträge zur Bildwissenschaft, hrsg. von Klaus Sachs-Hombach, Klaus Rehkämper, Springer, 2004.

„Interview mit Navid Kermani am 21.06.2016 in Köln“. Navid Kermani, hrsg. von Michael Hofmann, Klaus Stosch, Swen Schulte Eickholt, Königshausen \& Neumann, 2019, S. 251 f.

Kermani, Navid. Ungläubiges Staunen. Über das Christentum. Aufl. 14, Beck, 2017.

Krüger, Malte Dominik. Das andere Bild Christi. Mohr Siebeck, 2017.

Laak, Lothar van. Hermeneutik literarischer Sinnlichkeit. Historisch-systematische Studien zur Literatur des 17. und 18. Jahrhunderts. Max Niemeyer, 2003.

Langenhorst, Georg. ,Ich gönne mir das Wort Gott.' Annäherungen an Gott in der Gegenwartsliteratur. Herder, 2009.

Langenhorst, Georg. „'Sprachkrise im Theotop“. Von den Schwierigkeiten und Möglichkeiten. Gott zu Sprache zu bringen“. Rundbrief, Nr. 1, 2017, S. 11-16.

Latour, Bruno. Jubilieren. Über religiöse Rede. Aus dem Französischen Achim Russer. Suhrkamp, 2011.

Maffei, Lamberto, und Adrianna Fiorentini. Das Bild im Kopf: von der optischen Wahrnehmung zum Kunstwerk. Aus dem Italienischen von Dietmar Zimmer. Springer, 1997.

Markowski, Paweł: „Ekphrasis“. Pamiętnik literacki, Nr. XC,2, 1999, S. 229-232.

Motté, Magda. „Die Rede von Gott in der modernen Literatur.“ Spuren zum Geheimnis. Theologie und moderne Literatur im Gespräch, hrsg. von Thomas Schreijäck, Schwabenverlag, 2000, S. 13-52.

Müller, Julian; Grizelj, Mario. „Ein katholischer Tonfall? Michael Certeaus und Bruno Latours Zugänge zur religiösen Rede als Alternativen zu einer Intellektuellensoziologie“. Zeitschrift für Religion, Gesellschaft und Politik: Journal for Religion, Society and Politics, Nr. 3,1, 2019, S. 177-198.

Navid Kermani, Religion, Traditionsverlust und Fundamentalismus (NZZ Standpunkte 2015). https://www.youtube.com/watch?v=bvM1Td4HpIM. Abruf am 24.3.2020.

Rosen, Valeska. „Die Energeia des Gemäldes. Zu einem vergessenen Inhalt des Ut-pictura-poesis und seiner Relevanz für das cinquecenteske Bildkonzept". Marburger Jahrbuch für Kunstwissenschaft, Nr. 27, 2000, S. 171-208.

Sachs-Hombach, Klaus, und Klaus Rehkämper. Bild - Bildwahrnehmung - Bildverarbeitung. Interdisziplinäre Beiträge zur Bildwissenschaft. 2. Aufl., Deutscher Universitätsverlag, 2004.

Schmitz-Emans, Monika. Die Literatur, die Bilder, das Unsichtbare: Spielformen literarischer Bildinterpretation vom 18. bis 20. Jahrhundert. Königshausen und Neumann, 1999.

Strehle, Samuel. „Hans Belting: ,Bildanthropologie“ als Kulturtheorie der Bilder.“ Kultur. Theorien der Gegenwart, hrsg. von Stephan Moebius, Dirk Quadflieg. VS Verlag für Sozialwissenschaften, 2011, S. 507-518.

Wackenroder, Wilhelm Heinrich, und Ludwig Tieck. Herzensergießungen eines kunstliebenden Klosterbruders. Berlin, Johann Friedrich Unger, 1797. 


\section{„ZAŁÓŻMY, ŻE NIE ZNASZ TYTUŁU OBRAZU”: OBRAZY SAKRALNE W TEKSTACH NAVIDA KERMANIEGO}

\section{Streszczenie}

Wydaną w 2015 r. książkę Navida Kermaniego Ungläubiges Staunen. Über das Christentum można odczytać przede wszystkim jako próbę wykreowania nowego języka religijnego i powstrzymania dominującej we współczesnej literaturze marginalizacji religii jako zjawiska wyłącznie historycznego, estetycznego lub ekskluzywnego. Ta tendencja, określana przez Bruno Latoura mianem ikonoklazmu, prowadzi do zaniku umiejętności opisywania doświadczeń religijnych. Kermani odwołuje się w swoich refleksjach na temat obrazów sakralnych głównie do malarskiej tradycji katolickiego baroku i tworzy strategię pisarską, która ma na celu uwolnienie ich od dominujących obecnie interpretacji zarówno konfesyjnych, jak i świeckich. W przekraczaniu granicy między obydwoma mediami - obrazem i tekstem - i traktowaniu ich w sposób łączny upatruje on możliwość dotarcia do nieczytelnego dla współczesnego odbiorcy przeżycia religijnego oraz odtworzenia go w języku zakorzenionym w świecie jego doświadczeń, wyobrażeń i emocji.

Słowa kluczowe: Navid Kermani; obraz i słowo; intermedialność; literatura niemieckojęzyczna; język religijny.

\section{“SUPPOSING YOU WERE UNAWARE OF THE PAINTING'S TITLE": NAVID KERMANI AND SACRAL PAINTINGS}

\section{S u m mary}

Navid Kermani's Ungläubiges Staunen. Über das Christentum (2015) should be primarily understood as an attempt to create a new religious language and to contain the dominant literary tendency, which is to marginalise religion as a solely historical, aesthetic or exclusive phenomenon. This tendency, termed "iconoclasm" by Bruno Latour results in the disappearance of the ability to describe religious experiences. Kermani mainly refers to the tradition of Catholic baroque art when reflecting on sacral paintings, and invents a literary strategy that aims to free them from the prevalent interpretations, both confessional and secular. He considers the crossing of the boundaries between the two media - image and text - and their being treate as one as a possible way to reach that religious experience which is unintelligible to contemporary readers, and to recreate this experience in a language which rooted in a world of their own experiences, imagination and emotions.

Keywords: Navid Kermani; word and picture; intermediality; German literature; religious language. 